\title{
EXPLORANDO A ESPIRAL DE ARQUIMEDES COM SOFTWARE DE GEOMETRIA DINÂMICA
}

\author{
Edcarlos Vasconcelos da Silva - UNIFAP - edcarllosvasconcellos@ hotmail.com \\ José Carlos Pinto Leivas - UNIFRA - leivasjc@ unifra.br \\ Mariana Lopes Dal Ri - IFRR - marianadalri@yahoo.com.br
}

\begin{abstract}
Resumo: Este artigo apresenta análise e discussão de uma investigação realizada com estudantes do Ensino Médio na cidade de Macapá, no Estado do Amapá. Teve como objetivo explorar a construção da Espiral de Arquimedes no ambiente do software Geogebra e desenvolver a atividade de calcular o comprimento e o número de voltas de papel constantes de uma bobina utilizada para armazená-lo. A metodologia utilizada foi a investigação matemática e, para realizá-la, os investigadores planejaram e aplicaram uma sequência de passos orientadores para a resolução, a qual teve como instrumento de apoio o Geogebra. Os resultados da investigação mostraram que os alunos investigados conseguiram modelar uma bobina e calcular o número de voltas de papel que a mesma contém, bem como o seu comprimento. Por sua vez, os depoimentos dos estudantes mostraram que a utilização da metodologia e do software foram incentivadores para a aprendizagem e sugeriram ao professor titular empregá-la em outros conteúdos.
\end{abstract}

Palavras-chaves: Espiral de Arquimedes; Investigação Matemática; Geogebra.

\section{EXPLORING THE ARCHIMEDEAN SPIRAL WITH DYNAMIC GEOMETRY'S SOFTWARE}

\begin{abstract}
This article presents analysis and discussion of a research conducted with high school students in the city of Macapá, State of Amapá. The objective was to explore the construction of the Spiral of Arquimedes in Geogebra software and develop the activity of calculating the length of paper and the numbers of turns in a coil used to store it. The methodology used was the mathematical investigation and, to make it, the researchers prepared and applied a serie of guiding steps for the resolution, which had Geogebra as an instrument of support. The research results showed that the investigated students were able to model a coil and calculate the number of turns of paper that it contains, as well as its length. Besides, the students' testimony showed that the use of both the methodology and the software were motivators for the learning process, and suggested to the teacher he use it in other contents.
\end{abstract}

Keywords: Archimedean Spiral; Mathematical Investigation; Geogebra.

\section{Introdução e justificativas}

Na pesquisa em Matemática, principalmente no que diz respeito ao ensino, é cada vez mais comum o uso de softwares como recurso facilitador de aprendizagem. Nesse sentido, encontramos o Geogebra, software de geometria dinâmica, como um dos aplicativos mais difundidos no meio acadêmico atualmente, assim como em ambientes de formação e aperfeiçoamento de professores de Matemática. Isso se deve ao fato de ser totalmente gratuito e permitir o desenvolvimento de conceitos de geometria, álgebra e cálculo diferencial e integral, podendo ser facilmente explorado pelo usuário. Nesse contexto, ele vai ao encontro de uma proposta baseada na investigação matemática, pois cria um ambiente favorável à elaboração e exploração de conceitos em Matemática.

A tecnologia digital disponibiliza ferramentas interativas que criam objetos dinâmicos e manipuláveis. O conhecimento absorvido pelos alunos é muito mais 
expressivo com o uso de mídias do que comparado ao processo estático abordado por livros e quadro negro. A assimilação de conceitos e conjecturas é melhor demonstrada com desenhos em movimento, melhorando o processo de aprendizagem e o desenvolvimento cognitivo dos educandos. (VARGAS e NOTARE, 2014)

Oliveira et al. (2014, p. 4) ressaltam um diferencial do Geogebra como sendo o seu sistema, pois este "permite que o usuário realize construções e insira equações e coordenadas que estão diretamente interligadas. Logo, se fizer alguma alteração na construção geométrica, isso implicará em alterações nas representações algébricas."

Quanto à relevância para o uso da investigação matemática, entre outras metodologias, Corradi (2011) ressalta que argumentar e provar são alguns dos principais objetivos educacionais do ensino da Matemática. Eles se destacam nas atividades de investigação, uma vez que se caracterizam por situações em que os alunos tentam compreender, descobrir relações, semelhanças e diferenças de forma, para conseguirem chegar 0 generalizações que, posteriormente, serão confrontadas, assim como suas diferentes conjecturas e justificativas.

A investigação descrita e analisada no artigo teve como objetivo explorar a construção da Espiral de Arquimedes no ambiente do software Geogebra e desenvolver a atividade de calcular o comprimento e o número de voltas de papel constantes de uma bobina utilizada para armazená-lo. Isso se preenche de importância, uma vez que a Espiral de Arquimedes foi o ponto de partida para muitos estudos em cálculo diferencial e integral, além de possuir muitas aplicações, no desenvolvimento da Matemática e no próprio cotidiano.

\begin{abstract}
A matemática grega tem sido descrita como essencialmente estática, com pouca consideração pela ideia de variabilidade; mas Arquimedes, em seu estudo da espiral, parece ter achado a tangente a uma curva por considerações cinemáticas aparentadas ao cálculo diferencial. Pensando em um ponto sobre a espiral $r=a \theta$ como sujeito a um duplo movimento - um movimento radial uniforme, afastando-se da origem das coordenadas e um movimento circular uniforme em torno da origem - ele parece ter achado (através do paralelogramo de velocidade) a direção do movimento (logo, da tangente à curva) observando a resultante dos dois movimentos componentes. Parece ser esse o primeiro caso em que foi achada a tangente a uma curva que não era o círculo. (BOYER e MERZBACH, 2012, p. 102)
\end{abstract}

Daí darmos também importância ao estudo da espiral de Arquimedes nesta investigação. Essa curva possibilita subdivisões de ângulos, como também quadrar o círculo, como Arquimedes mostrou.

\title{
2. A Espiral de Arquimedes
}

Compreendemos curvas planas como aquelas que estão contidas em um único plano contido no espaço euclidiano; as quais podem ser abertas como as parábolas, ou fechadas como as circunferências, por exemplo. Tais curvas possuem diversas representações: formas algébricas, paramétricas (ou polares) e geométricas (gráficas). Nos dois primeiros aspectos a janela de álgebra do Geogebra e a de entrada desempenham papel tão fundamental quanto a de visualização para a compreensão e associação do objeto curva. Elas são importantes no tratamento de comprimentos, áreas, flexão, torção, etc.

Segundo Gercov e Caputi (2008), desde os primórdios da geometria, as curvas são objetos de estudos e fascinação dos matemáticos. Ao longo do tempo eles buscaram classificá-las, medi-las e modelá-las, tendo em vista que elas possuem importantes 
aplicações na vida real, especialmente no que tange a fenômenos naturais estudados pela Física, Química e Biologia.

\begin{abstract}
A humanidade sempre foi fascinada pelas curvas muito antes de estas serem vistas como objetos matemáticos. Uma prova disso são os caminhos de ondas e espirais em cerâmica pré-histórica, ou as dobras localizadas nas esplêndidas esculturas gregas e góticas. Foram os geômetras gregos que iniciaram o estudo de curvas definindo-as geometricamente como, por exemplo, o contorno da intersecção de um plano com um cone. A linha e o círculo eram como bordas distintas de seções planas de um cone (GARCIA, 2007, p. 08).
\end{abstract}

De maneira intuitiva, Alencar e Santos (2003, p. 11) assim definem curvas planas: "uma deformação contínua de um intervalo, ou ainda, a trajetória de um deslocamento de uma partícula no plano". Funções de uma única variável real definidas num subconjunto de números reais podem ter seu gráfico desenhado em uma folha de papel [uma representação de um plano euclidiano] com um único traço e constituem uma representação geométrica plana.

Um grupo muito especial dessas curvas, denominado de Curvas Planas Notáveis, devido às propriedades que apresentam, é constituído de: rosácea; cardioide; lemniscata; cicloide; astroide; cissoide; espiral de Fermat; e espiral de Arquimedes. Neste artigo, nosso foco de estudo pertence à última delas, na qual apresentamos algumas propriedades e um modo de explorá-la em sala de aula de forma interativa, com uso de geometria dinâmica.

A Espiral de Arquimedes foi estudada pelo matemático da Antiguidade Clássica Arquimedes de Siracusa (287 - 212 a.C.) na obra Sobre as Espirais, na qual ele define a espiral do ponto de vista físico como sendo o "lugar geométrico dos pontos $P$ de uma reta que gira em torno do centro $O$ com velocidade angular constante e o ponto $P$ se desloca sobre a reta $r$ a partir de $O$ com velocidade constante sobre a reta" (ROSO; CLAUDIO; CANALLI, 2006, p. 02).

Arquimedes mostrou, por meio de sua espiral, que era possível fazer a trissecção de um ângulo sem muito esforço, resolvendo um problema matemático que intrigava estudiosos da Grécia Antiga. Eles tinham fascinação por desafios e a divisão de um ângulo arbitrário, em três partes iguais, sem utilizar instrumentos de medição, foi um problema que movimentou a matemática antiga. A figura 1 ilustra a espiral de Arquimedes.

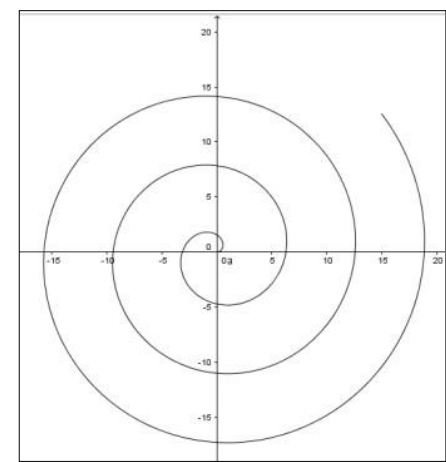

Figura 1 - A espiral de Arquimedes Fonte: autores

O estudo da espiral, assim como das outras curvas citadas, pode incentivar os alunos a pesquisar e aumentar o interesse deles em investigação alicerçada no uso de geometria dinâmica, pois eles terão a oportunidade de compreendê-la e definir alguns conceitos importantes, a partir de suas construções; no caso presente, com o software de geometria dinâmica. 


\title{
3. Geometria dinâmica e o software Geogebra
}

Diversos programas utilizados para se ensinar geometria são dinâmicos e abordam uma representação semiótica. Diversas formas e objetos são manipulados na tela do computador. Desta forma, um objeto geométrico abstrato torna-se visível, criando ou potencializando o raciocínio geométrico do aluno, assim como uma sintonia entre os conceitos individuais e os significados geométricos da matemática teórica. (SOUZA e GRAVINA, 2009)

O termo geometria dinâmica é usado para ressaltar a diferença entre os softwares de geometria. Os de geometria dinâmica diferem dos demais por possuírem um recurso que permite a transformação contínua em tempo real. Em geral, associada ao uso do computador, ela se refere à geometria dos softwares que permitem a construção de figuras que podem ser arrastadas pela tela, em tempo real, por movimentos no cursor. Esse movimento é controlado pelo usuário e preserva os vínculos estabelecidos na construção.

Zullato (2002), em sua pesquisa, ressalta que o uso de geometria dinâmica pelos professores, em sala de aula de Matemática, permite atividades de livre exploração, usualmente aliada ao computador, em um ambiente próximo ao que ele já conhece, porém com mais possibilidades do que a mídia tradicional (lápis e papel).

\begin{abstract}
No que tange à exploração, o aluno pode formular suas próprias conjecturas e tentar verificar se elas são válidas. Ou seja, o próprio aluno irá realizar a verificação e validação da conjectura que formulou. Isso é possível devido aos recursos dos softwares, como o arrastar, que possibilita a simulação de diferentes casos da figura, como se o aluno estivesse verificando "todos" os casos possíveis de uma mesma família de configuração. (ZULLATO, 2002, p.21)
\end{abstract}

Nesse contexto, nossa investigação foi alicerçada no uso do Geogebra, o qual pode ser facilmente instalado em qualquer computador ${ }^{1}$. No website também podem ser encontrados diversos materiais, que apresentam seu uso em diferentes áreas da Matemática, nos mais variados contextos, aplicações e apresentações. A figura 2 corresponde a essa página, apresenta o aplicativo como uma calculadora gráfica para funções como geometria, álgebra, cálculo, estatística e matemática 3D. A figura 3, também disponível no mesmo endereço, ilustra essas aplicações.

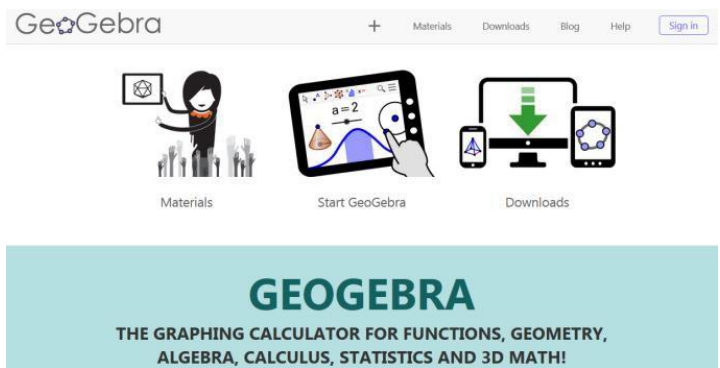

Figura 2 - Pagina inicial do Geogebra.

Fonte: http://www.geogebra.org/

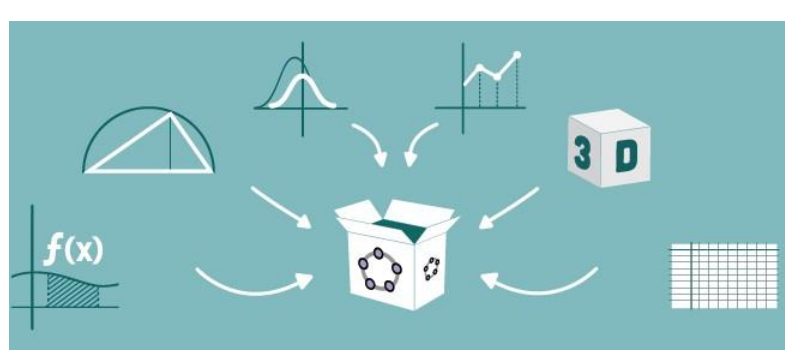

Figura 3 - Aplicações do Geogebra. Fonte: http://www.geogebra.org/download

O Geogebra é um software livre e gratuito; está disponível em diversos idiomas e funciona em diferentes plataformas. Seu criador foi Markus Hohenwarter, que o concebeu durante um curso de mestrado na Universidade de Salzburgo (Austria), para ser usado de forma intuitiva por usuários sem conhecimentos aprofundados de informática. Como sua aplicação está voltada à Matemática Dinâmica, ele permite a realização de trabalhos em

${ }^{1} \mathrm{O}$ download está disponível em http://www.geogebra.org/cms/pt_BR/info . 
diferentes níveis estudantis, representando objetos tanto em forma geométrica quanto algébrica. (HOWENWARTER, 2007, apud MOREIRA et al., 2013)

Corrêa Jr. e Raab (2015), com o objetivo de avaliar de que forma o Geogebra pode contribuir para o desenvolvimento dos estudantes de Matemática, no que diz respeito à percepção de padrões, relataram, em sua pesquisa, que o software permitiu uma abordagem diferenciada. Os autores afirmam que ele proporcionou aos alunos uma experiência diferente da habitual com a disciplina de Matemática, assim como um aprendizado mais significativo, por permitir a exploração e investigação de novas possibilidades. No que se refere à educação matemática, os autores ressaltam que "é fundamental que se investigue e se busquem alternativas para tornar os processos de aprender e ensinar Matemática mais eficientes e eficazes" (p. 8). Com isso, é possível perceber a importância da investigação em Matemática, em um ambiente como o Geogebra, propicio para estudar, verificar e provar teoremas.

Nesse contexto, a seguir, apresenta-se o percurso realizado para a elaboração da investigação, seguida das atividades elaboradas e aplicadas para realização no ambiente Geogebra, bem como os conceitos que os investigadores pretenderam alcançar.

\section{Abordagem metodológica}

A pesquisa teve início em uma disciplina de mestrado, em que foram exploradas diversas ferramentas do software Geogebra, a fim de responder ao seguinte questionamento: de que forma poderemos utilizar o software Geogebra para desenvolver uma atividade motivadora para o estudo da Espiral de Arquimedes?

Entende-se que a investigação realizada apresenta uma abordagem metodológica interpretativa, no sentido apontado por Moreira (2011), na qual "o pesquisador interpretativo registra eventos, obtém dados, transforma-os e faz asserções." (p. 50). Além disso, "O investigador interpretativo observa participativamente, de dentro do ambiente estudado, imerso no fenômeno de interesse, anotando cuidadosamente tudo o que acontece nesse ambiente, registrando eventos." (p.50)

Para o procedimento de coleta de dados, os investigadores, imersos no processo de construção dos estudantes no ambiente informatizado, realizaram registros escritos de observações próprias, as quais aconteceram no transcorrer da realização das atividades, bem como recolheram as tarefas executadas por meio digital. Dessa forma, a análise a posteriori pode ser feita em conjunto pelos três pesquisadores, de modo a obter as conclusões que são anunciadas no artigo.

A partir da questão de pesquisa foi realizado um levantamento bibliográfico que fundamentasse uma proposta de atividades a serem investigadas em sala de aula pelos investigadores. Foi elaborado um roteiro dessas atividades, para serem exploradas no ambiente Geogebra, baseado na metodologia de investigação matemática. Como objetivo específicos da investigação foram propostos: explorar a Espiral de Arquimedes; definir alguns conceitos relevantes acerca do tema; buscar despertar nos alunos uma postura participativa e investigativa na aula.

Os Parâmetros Curriculares Nacionais ressaltam que o ensino da Matemática "prestará sua contribuição à medida que forem exploradas metodologias que priorizem a criação de estratégias, o espírito crítico, autonomia e confiança na capacidade de enfrentar desafios" (BRASIL, 1998, p. 26). Nesse sentido, Corradi (2011), conclui:

[...] a relevância dessa atividade se deve ao desenvolvimento do trabalho que é realizado em equipe, onde a utilização da argumentação, da comunicação matemática e da elaboração de relatórios, oportuniza aos alunos a produção de significados para a Matemática. Através de situações problema desafiadoras 
possibilita ao aluno o desenvolvimento de autonomia na busca de meios para investigação. (CORRADI, 2011, p. 5 ou 165)

Além disso, a autora destaca em seu trabalho, considerações de vários autores, (Rocha e Ponte (2006), Cunha, Oliveira e Ponte (1995), Fonseca, Brunheira e Ponte (1999), Mendes (1997), Brocardo (2002), apud CORRADI, 2011) que, de um modo geral, concordam que propostas de investigação ajudam a desenvolver a capacidade de criação de soluções e de um ambiente de trabalho estimulante para a aprendizagem, pois, para compreender a Matemática, é essencial que se compreenda também sua construção.

No que se refere ao roteiro de intervenção, buscou-se explorar o cálculo do diâmetro e do comprimento linear de uma espiral. A atividade em questão consiste em desenhar uma bobina-espiral no ambiente do software Geogebra e calcular o comprimento linear $M$ e seu número de voltas $n$ com base nos parâmetros coletados no aplicativo.

Os alunos participantes da pesquisa, trabalhando em duplas, no laboratório de informática da escola em que foi aplicada a atividade, receberam as instruções (passo-apasso) acerca do que deveriam construir, assim como retomada do aplicativo do qual já tinham conhecimentos. Foram orientados a salvarem suas construções, pois as mesmas seriam recolhidas dos computadores para a análise e produção de um artigo científico, e que suas identidades seriam preservadas. Participaram da investigação doze estudantes do terceiro ano do ensino médio de uma escola pública estadual da Cidade de Macapá, no Estado do Amapá.

Os investigadores, de início, propuseram: de que forma poderemos utilizar o software Geogebra para desenvolver uma atividade motivadora para o estudo da Espiral de Arquimedes? A fim de respondê-la foi lançado o seguinte problema: como calcular o número de voltas de papel contido em uma bobina e seu comprimento total. A partir disso, a atividade concentrou-se em levar os alunos a produzirem uma simulação no ambiente do software Geogebra e, com isso, calcular o comprimento linear $\mathrm{M}$ do papel e o número de voltas $n$ na bobina seguindo as seguintes etapas:

1) Abrir o software Geogebra e construir a Espiral de Arquimedes utilizando os comandos fornecidos pelos professores-pesquisadores;

2) Utilizar o software Geogebra para aproximar a Espiral de Arquimedes a uma bobina de papel comum.

3) Retirar numericamente os seguintes parâmetros da espiral: raio maior $R$, raio menor $r$, espessura $e$ do material e diâmetro $D$.

4) Calcular o número de voltas utilizando a equação: $D=2(r+e n)$

5) Calcular o número $M$ do comprimento linear da bobina, utilizando a equação: $\pi e n 2+(2 \pi r+\pi e) n-M=0$.

No decorrer da investigação, os estudantes observavam e manipulavam suas construções, sendo constantemente convidados a relatarem suas observações e até mesmo registrá-las por escrito para posterior discussão com os demais colegas no grande grupo.

Por fim, foi formalizado, pelos investigadores, o conhecimento explorado na atividade, definindo conceitos acerca do tema proposto, com base na literatura e que constam do quadro1.

Quadro 1- Alguns conceitos envolvidos no trabalho

\begin{tabular}{|l|l|}
\hline \multicolumn{1}{|c|}{ Conceito } & \multicolumn{1}{c|}{ Definição } \\
\hline Curva Plana & $\begin{array}{l}\text { Uma deformação contínua de um intervalo, ou ainda, a } \\
\text { trajetória de um deslocamento de uma partícula no plano. }\end{array}$ \\
\hline
\end{tabular}




\begin{tabular}{|l|l|}
\hline Espiral de Arquimedes & $\begin{array}{l}\text { Lugar geométrico dos pontos } P \text { de uma reta que gira em } \\
\text { torno do centro } O \text { com velocidade angular constante e o } \\
\text { ponto } P \text { se desloca sobre a reta } r \text { a partir de } O \text { com } \\
\text { velocidade constante sobre a reta. }\end{array}$ \\
\hline Comprimento Linear $M$ & Comprimento total da Espiral de Arquimedes. \\
\hline Total de voltas $n$ & $\begin{array}{l}\text { Número total de voltas no eixo central que a Espiral de } \\
\text { Arquimedes produz. }\end{array}$ \\
\hline
\end{tabular}

Fonte: Georges (2010), Alencar e Santos (2003)

\section{Análise dos resultados}

Quando os alunos foram questionados sobre o conhecimento que tinham acerca do conteúdo em foco, suas respostas se limitaram a gestos de giros nos dedos, indicando o formato de uma espiral, o que foi registrado pelos investigadores. Eles realizaram a primeira etapa sem dificuldade. Construíram a espiral aplicando a equação da espiral no Geogebra, fornecida pelos investigadores, na janela de entrada do software. De imediato, observaram na janela de visualização o traçado da curva (Figura 4, A).

$\mathrm{Na}$ segunda etapa, em sequência, os alunos aproximaram a espiral para uma bobina no próprio Geogebra e, ainda, demonstraram utilizar a criatividade ao trabalhar com as ferramentas círculo e ponto para desenharem o modelo da bobina sobreposto à espiral obtida pela equação fornecida (conforme ilustrado na Figura 4, B), a qual foi salva e encaminhada aos pesquisadores.

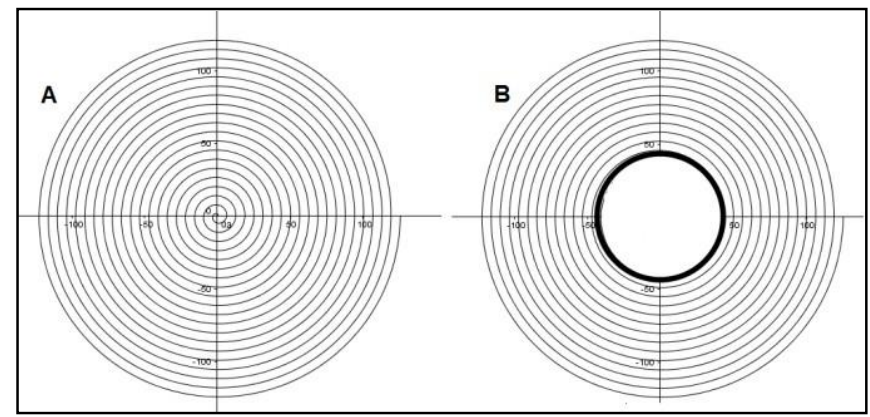

Figura 4 - Espiral de Arquimedes e simulação de uma bobina. Fonte: construção realizada por uma dupla de alunos.

Para obter os parâmetros, tarefa solicitada na terceira etapa, os alunos utilizaram os recursos do Geogebra: reta, segmento de reta, medir os raios e a espessura da espiral. Foi sugerido que utilizassem uma tabela e, a partir daí, completassem com os dados coletados. Por fim, de posse dos parâmetros foi possível calcular o número de voltas $n$ da bobina, bem como seu comprimento total $M$.

Ressalta-se aqui que os alunos exploraram livremente o software e utilizaram sua curiosidade para procurar os recursos disponíveis, de forma a não depender de orientação constante dos professores-pesquisadores. Com isso, a postura investigativa que se esperava dos alunos foi alcançada.

Após a realização das atividades no Geogebra, os alunos foram convidados a discutir suas interpretações, retomar a questão inicial do que entendiam por espiral, elaborar conclusões e definições informais.

Alguns alunos relataram que não imaginavam poder usar o computador para ilustrar o cálculo do número de voltas em uma bobina, como os seguintes: - eu não sabia que era assim que se calculava a distância total do papel de um rolo de bobina (aluno A); - eu também não pensava que dava pra calcular o número de voltas que o papel dá 
ao redor do "cano preto" da bobina, isso foi muito legal (Aluno B); - o programa do computador (Geogebra) foi bom pra fazer a gente ver como faz (Aluno C).

Durante a execução da atividade notou-se o empenho dos alunos para chegarem logo à solução do problema. Na sequência foram levantadas outras considerações e retomados os conceitos iniciais previstos na investigação. Após a realização dessa atividade final, foi possível perceber o interesse dos alunos por aulas com uso de aplicativos. Perguntaram sobre novos assuntos que poderiam ser desenvolvidos com as ferramentas disponíveis no Geogebra e se poderiam voltar ao laboratório de informática.

\section{Considerações finais}

A partir da investigação realizada foi possível verificar que o software Geogebra foi um instrumento facilitador do processo de aprendizagem. As atividades realizadas cumpriram com os objetivos de explorar a construção da Espiral de Arquimedes no ambiente do software Geogebra e desenvolver a atividade de calcular o comprimento e o número de voltas de papel constantes de uma bobina utilizada para armazená-lo. O aplicativo permitiu que todos os alunos elaborassem suas construções, análises e verificações, atingindo o objetivo deste trabalho. Dessa maneira, conceituar e compreender geometria se tornou algo muito mais palpável e concreto, permitindo que o grupo de estudantes envolvidos na investigação, colocasse à prova diferentes hipóteses, teoremas, conjecturas, fórmulas e axiomas.

Ressalta-se que o trabalho com geometria dinâmica é uma atividade totalmente livre, onde os alunos são convidados a realizarem suas construções, explorarem o ambiente e levantarem inúmeras questões acerca de um tema proposto. Entretanto, é de suma importância a mediação do professor na elaboração de questões que permitam e facilitem a aprendizagem. Perceber quando é necessário intervir, para que o trabalho em sala de aula não perca o foco é provavelmente um dos momentos mais decisivos; por isso o professor precisa estar atento, tanto à elaboração dos objetivos, quanto aos passos (questões, discussões, proposições, etc) que surgem durante a aula para que os objetivos sejam, de fato, alcançados.

$\mathrm{Na}$ investigação, objeto do presente artigo, os alunos concluintes do Ensino Médio, público alvo das atividades, já tinham um breve conhecimento do aplicativo, pois de antemão já conheciam alguns comandos e já haviam explorado o software em outras ocasiões. O laboratório de informática da escola também já estava preparado, com a instalação do Geogebra. Esses são alguns dos fatores que devem ser levados em consideração para a aplicação de uma proposta com uso de geometria dinâmica, o que pode proporcionar resultado positivo. Além disso, o texto sugere a exploração do software não somente em geometria, mas também em álgebra, cálculo, estatística e matemática 3D. Outras propostas podem ser encontradas no website do Geogebra, que por ser de fácil manuseio e até um tanto quanto intuitivo, os professores que tiverem interesse em utilizálo podem elaborar atividades mais completas e/ou inovadoras em suas aulas.

Os alunos adquiriram autonomia e motivação, especialmente a partir da questão motivadora levantada, para a realização das atividades e também sugeriram outras que poderiam ser realizadas na própria disciplina em desenvolvimento. Essas atividades investigativas permitiram ao professor, em outras ocasiões, trabalhar com alunos de diferentes níveis de desenvolvimento e fez com que ele se sentisse estimulado a repensar sua prática docente. $\mathrm{O}$ mais interessante é que os resultados podem ser muito variados e as discussões muito ricas e, como já afirmaram Ponte, Brocardo e Oliveira (2009), em uma aula de investigação é sempre possível programar o modo como ela vai começar, mas nunca se sabe como ela irá acabar. 
Os investigadores pretendem aprofundar a pesquisa, em ações futuras, nas quais o uso do Geogebra 3D pode ser utilizado para o cálculo de fios em outras formas de bobinas, como por exemplo, no cálculo da indutância em um circuito, para a qual um dos fatores é o comprimento dos condutores (conforme ilustrado na Figura 5).

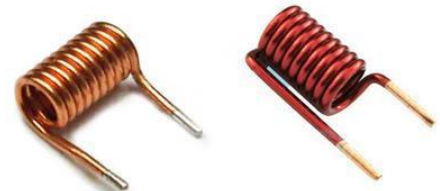

Figura 5 - Bobina

Fonte: http://blog.render.com.br/tutorial-2/como-medir-a-indutancia-das-bobinas/

Referências

ALENCAR, H.; SANTOS, W. Geometria Diferencial das Curvas Planas. In: Colóquio Brasileiro de Matemática, 24., 2003, Rio de Janeiro. Anais. Rio de Janeiro: IMPA. Disponível em: <http://goo.gl/vfVKmw> Acesso em: 01 mai. 15.

BOYER, C. B.; MERZBACH, U. C. História da Matemática. São Paulo: Blucher, 2012. $504 \mathrm{p}$.

BRASIL. Ministério de Educação. Secretaria de Ensino Fundamental. Parâmetros Curriculares Nacionais: Matemática, Ensino de Quinta a Oitava séries Brasília, 1998. Disponível em: <http://portal.mec.gov.br/seb/arquivos/pdf/matematica.pdf> Acesso em: 20 fev. 2015.

CORRADI, D. K. S. Investigações matemáticas. Revista da Educação Matemática da UFOP. Ouro Preto. Vol. 1, p. 162-175, 2011. Disponível em: <http://www.cead.ufop.br/jornal/index.php/redumat/article/view/346/303>. Acesso em: 15 mar. 2016.

CORRÊA JR., V. J.; RAAB, A. L. A. Uma experiências de uso do geogebra na identificação de padrões em trigonometria. Revista Tecnologias na Educação, ano 7, n.13, dez. 2015. Disponível em <http://tecnologiasnaeducacao.pro.br/wpcontent/uploads/2015/12/Art24-vol13-dez2015.pdf>. Acesso em: 15 mar. 2016.

GARCIA, M. C. F. Galeria de curvas no plano. Revista Electrónica de Contenido Matemático, Cidade do México, v.24, 2007. Disponível em: <http://www.redmat.unam.mx/foro/volumenes/vol024/MaterialDeApoyoEnMaple9.pdf >. Acesso em: 13 fev. 2015. Título original: Galeria de curvas en el plano.

GEOGEBRA 2016 - Matemática Dinâmica para aprender e ensinar. Disponível em: < http://www.geogebra.org/>. Acesso em: 20 abr. 2016. Título original: Geogebra 2016 - Dynamic Mathematics for learning and teaching.

GEORGES, M. R. R. Metodologia para cálculo do diâmetro e do comprimento de bobina de papel auto-adesivo. Revista Iberoamericana de Engenharia Industrial, Florianópolis, v. 02, n. 02, p. 108-123, 2010. Disponível em 〈http://goo.gl/D4O9SL〉 Acesso em 01 mar. 2015.

GERCOV, I. G.; CAPUTI, A. Geometria das Curvas Planas. In: SIMPÓSIO DE INICIAÇÃO CIENTÍFICA DA UFABC, 1., 2008, Santo André. Anais: Santo André: 
Centro de Matemática/Universidade Federal do ABC., 2008, p.24. Disponível em: <http://gradmat.ufabc.edu.br/iniciacao/2008/sicufabc2008_submission_24.pdf> Acesso em 10 jan. 2015.

MOREIRA, L. S.; PEIXOTO, G. T. B.; BATISTA, S. C. F. Geomêtria Dinâmica em tablets: estudo de caso com o aplicativo Geogebra. Revista Renote: Novas Tecnologias na Educação, Porto Alegre, v. 11, n. 3, p. 1-10, 2013; Disponível em: <http://seer.ufrgs.br/index.php/renote/article/view/44715/28449>; Acesso em: 22 mar. 2016.

MOREIRA, M.A. Metodologias de pesquisa em ensino. São Paulo: Editora Livraria da Física, 2011.

OLIVEIRA, S. S.; OLEQUES, N. M. B. N.; ROCHA, I. B. P.; OLIVEIRA, C. P. Geogebra: As novas tecnologias aplicadas ao ensino da matemática. In: ESCOLA DE INVERNO DE EDUCAÇÃ̃ MATEMÁTICA, 4., 2014. Santa Maria. Anais. Santa Maria: UFSM. Disponível em <http://w3.ufsm.br/ceem/eiemat/Anais/arquivos/ed_4/RE/RE_sabedra_sersana.pdf > Acesso em 20 de março de 2016.

PONTE, J. P.; BROCARDO, J.; OLIVEIRA, H. Investigações matemáticas na sala de aula. Belo Horizonte: Autêntica Editora, 2009.

ROSO, D. S.; CLAUDIO, D. M.; CANALLI, V. Introdução ao modelamento da Espiral de Arquimedes para aplicação em sistemas hidráulicos. In: SALÃO DE INICIAÇÃO CIENTÍfICA, 6., 2006, Porto Alegre. Anais. Porto Alegre: Seção de Ciências Exatas e da Terra/PUC-RS, 2006. Disponível em: <http://www.pucrs.br/research/salao/2006VIISalaoIC/Arquivos2006/CienciasExatasedaTerra/36981\%20-

\%20DANIEL\%20SIGNORI\%20ROSO.pdf> Acesso em 13 fev. 2015.

SOUSA, J. M. R. Trissecção do ângulo e duplicação do cubo: as soluções na Antiga Grécia. Porto: DMP/FCUP, 2001. 113p. Dissertação de mestrado. Disponível em: <http://rodrigomat2004.pbworks.com/w/file/fetch/89736425/3_problemas_classicos.pdf $>$ Acesso em: 01 mar. 2015.

SOUZA, C. E.; GRAVINA, M. A. Geometria com animações interativas. Revista Renote: Novas Tecnologias na Educação, Porto Alegre, v. 7, n. 1, p. 1-9, 2009; Disponível em: <http://seer.ufrgs.br/index.php/renote/article/view/13999/7888>; Acesso em: 18 mar. 2016.

VARGAS, E. L.; NOTARE, M. R. Integração de diferentes mídias digitais no ensino de Geometria: uma experiência com o oitavo ano do ensino fundamental; Revista Renote: Novas Tecnologias na Educação, Porto Alegre, v. 12, n. 2, p. 1-10, 2014; Disponível em: <http://seer.ufrgs.br/index.php/renote/article/view/53535/33041>; Acesso em: 18 mar. 2016.

ZULLATO, R. B. A. Professores de matemática que utilizam softwares de geometria dinâmica: suas características e perspectivas. Rio Claro: IGCE/UEP, 2002. 316p. Dissertação de Mestrado. Disponível em <http://www.geogebra.imuff.mat.br/biblioteca/zulatto_rba_me_rcla.pdf> Acesso em: 15 mar. 2016. 